九州大学学術情報リポジトリ

Kyushu University Institutional Repository

\title{
Note on the Separation of Reacting System by Chromatography
}

Kuhara, Satoru

Laboratory of Sericultural Chemistry, Faculty of Agriculture, Kyushu University

Iwamoto, Shinichi

Laboratory of Sericultural Chemistry, Faculty of Agriculture, Kyushu University

Yanase, Yuji

Laboratory of Sericultural Chemistry, Faculty of Agriculture, Kyushu University

Fukamizo, Tamo

Laboratory of Sericultural Chemistry, Faculty of Agriculture, Kyushu University

他

https://doi.org/10.5109/23756

出版情報: 九州大学大学院農学研究院紀要. 27 (1/2)，pp.33-45，1982-10. Kyushu University バージョン：

権利関係 : 


\title{
Note on the Separation of R eacting System by Chromatography
}

\author{
Satoru Kuhara, Shinichi Iwamoto, Yuji Yanase, \\ Tamo Fukamizo and Katsuya Hayashi \\ Laboratory of Sericultural Chemistry, Faculty of Agriculture, \\ Kyushu University 46-02, Fukuoka 812
}

(Received May 27, 1982)

\begin{abstract}
The spatial distribution of substances in a reacting and diffusing system has attracted a special interest of many investigators in connection with the operation of immobilized enzyme column, mechanism of enzymatic reaction under cellular conditions and so on. In the present study, the experimental results on the separation of $\alpha$ - and $\beta$-anomers of 2-acetamido-2-deoxy-D-glucopyranose with a partition column chromatography were analyzed by computer simulation, as an example of reacting and diffusing system.
\end{abstract}

\section{INTRODUCTION}

The analysis of a reacting and diffusing (moving) system has been recently attracted many worker's interest in relation to the real aspect of biochemical system under cellular conditions and to the biotechnological operations. The exact description of dynamical behavior of biochemical system in living cells requires first the mathematical formulation of the system. Because of the heterogeneous nature of the inside of cell, the biochemical system should be regarded as reacting and diffusing system and should be formulated by suitable technique. The experimental results on the reacting and diffusing system, then, should be analyzed using the mathematical equation and at the same time the global nature of reacting and diffusing system should be surveyed by the computer simulation of the dynamics of the system using the equation. However, such an analysis appears to be rarely made, probably owing to the essential difficulty of the handling of the mathematical equation formulated for the reacting and diffusing system.

On the other hand, the reactions in an immobilized enzyme column have been analyzed in relation to the biotechnological aspect and the fruitful results have been obtained (Thomas and Kernevez, 1976). The fundamental differences between the cellular system and immobilized enzyme system are that the former contains several communicable phases in which many reactants are interacting each other. This situation of the cellular system makes it difficult to mathematically formulate the system and to solve the equation describing the dynamic behavior of the system.

In the present study, the behavior of a reversibly reacting system in a separation column, partition column, was investigated to establish the basic 
technique which can be applied to the analysis of the enzymatic reaction system under cellular conditions. Similarly to the general reducing sugars (Pigman and Anet, 1972), 2-acetamido-2-deoxy-D-glucopyranose mutarotates at a considerably high rate in aqueous medium. When this reversibly reacting system was applied on a partition column to separate each anomer, a specific elution pattern was observed owing to the reversible reaction. In the present study, the elution pattern was analyzed to estimate the values of the rate constants of the reversible reaction in the column and to predict the ratio of $\alpha$-anomer to $\beta$-anomer in the original solution which was applied on the column.

\section{MATERIALS AND METHODS}

\section{Materials}

$\beta$-Anomer of 2-acetamido-2-deoxy-D-glucopyranose, $\beta$-GlcNAc, was synthesized from 2-amino-2-deoxy $\rightarrow \alpha, \beta$-glucopyranose by the method of Kuhn and Haber (1953). $\{\alpha]_{D}^{2}\left(\mathrm{c}=0.5 \%, \mathrm{H}_{2} \mathrm{O}\right)$ of synthesized $\beta$-GlcNAc was -18.1" and the purity was calculated to be about $95 \%\left(\{\alpha]_{D}^{20}\right.$ of pure $\beta$-GlcNAc is -21.5”). This preparation showed a main second peak and a minor first peak on high-performance partition chromatography. Acetonitrile used for chromatography was of special grade.

\section{High-perfortiance partition chromatography}

High-performance partition. chromatography was carried out with a Jasco TRI ROTAR II. A column $(6 \times 500 \mathrm{~mm})$ of Shodex Ionpak S-614 was used. $16 \mu$ of sample soiution (about $1.5 \mathrm{mg} / \mathrm{ml}$ ) was applied on' the column and the elution was performed with $75 \%$ acetonitrile at flow rate of $0.3 \mathrm{ml} / \mathrm{min}$ (pressure; about $10 \mathrm{~kg} / \mathrm{cm}^{2}$ ) and at room temperature. The elution pattern was monitored by UV absorption measurement at $220 \mathrm{~nm}$. The concentration 'of each anomer was calculated from the area under the peak (Fukamizo and $\mathrm{H}$ a y a s h i, ' 1982 ).

\section{MATHEMATICAL A N A L Y S IS}

\section{State equation}

Partition column chromatography of reacting system involves three basic processes, the flows of eluent and solute along the vertical axis of the column, diffusions of the solute in vertical and lateral directions and reaction of the solutes in a column. The flow rate of eluent and solutes in liquid phase (outside of support phase) are same each other. However, the solutes diffuse in and from the support, and during diffusions the solutes stay for a period in the support in which the lateral flows of eluent and solutes do not occur. Such detour of solute causes the decrease in the overall flow rate of solute according to the partition coefficient of the solute.

In macroscopic analysis of partition chromatography, the column is divid- 
ed into a number of region which is called theoretical plate and it is assumed that in each plate equilibrium of solute between liquid phases is completely realized (Keller and Giddings, 1975). The distribution of solute in the plates was, found to be represented by the binominal expansion of $(1 / v-x)+x)^{\text {", }}$, where $\mathrm{x}$ is the solute fraction washed down to the next plate by a small volume of eluent and $n$ the number of theoretical plate and $v$ the volume per plate. It was shown that when $n$ is large the distribution will convert to Gaussian (Keller and Giddings, 1975).

In the present study, the vertical distribution of solutes were calculated microscopically on the basis of that the diffusion processes of solutes in liquid and support phases govern principally the distribution. The vertical component of diffusion in the both phases relates directly to the solute distribution. The lateral diffusion in liquid phase does not change the concentration of solute, and that in the support phase controls the solute transfer between the both phases, causing the delay of the flow and vertical distribution of solute. As described above, the solute distribution caused by lateral diffusion in the support phase will be represented by Gaussian. Thus, vertical distribution of solute in partition chromatography can be formulated by means of one-dimensional vertical diffusion.

2-Acetamido-2-deoxy-D-glucopyranose mutarotates rapidly in an aqueous medium. In homogeneous solution, each of reversible reactions in mutarotation can be represented by the first-order reaction (Fukamizo and Hayashi, 1982). In partition column, the rate constants of forward and reverse reactions in liquid phase may be different from those in support phase. However, it is very difficult to estimate the rate constants of mutarotation in support phase. In the present study, therefore, it was assumed that the rate constants in liquid and support phases are the same each other.

From the above consideration, the, separation process of mutarotating 2acetamido-2-deoxy-D-glucopyranose (GIcNAc) in partition column may be formulated as,

$$
\begin{aligned}
& \frac{\partial c_{\alpha}}{\partial T}=D_{\alpha} \frac{\partial^{2} c_{\alpha}}{\partial X^{2}}-v_{\alpha} \frac{\partial c_{\alpha}}{\partial X}-k_{1} c_{\alpha}+k_{2} c_{\beta}, \\
& \frac{\partial c_{\beta}}{\partial T}=D_{\sigma} \frac{\partial^{2} c_{\beta}}{\partial X^{2}}-v_{\beta} \frac{\partial c_{\beta}}{a X}+k_{1} c_{\alpha}-k_{2} c_{\beta},
\end{aligned}
$$

where $c_{\alpha}$ and $c_{\beta}$ are molar concentrations of a-anomer and $\beta$-anomer of GlcNAc at time $T, D_{\alpha_{0}}$ and $D_{\beta}$, the diffusion coefficient of anomers, $v_{\alpha}$ and $v_{\beta}$ the flow rate of anomers, $k_{1}$ and $k_{2}$ are the first-order rate constants of

$$
\alpha-\mathrm{GlcNAc} \underset{k_{2}}{\stackrel{k_{1}}{\rightleftharpoons}} \underset{-\mathrm{GlcNAc},}{ }
$$

and the vertical distance from the top of the column is represented by $\mathrm{X}$. The new dimensionless variables and parameters are defined as, 


$$
\begin{aligned}
& x=\frac{X}{L}, \quad t=\frac{v_{\alpha}}{L} T, C_{\alpha}=\frac{c_{\alpha}}{c_{\alpha}+c_{\beta}}, C_{\beta}=\frac{c_{\beta}}{c_{\alpha}+c_{\beta}}, \\
& K_{1}=\frac{L}{v_{\alpha}} k_{1}, \quad K_{2}=\frac{L}{v_{\alpha}} k_{2}, \Theta_{\alpha}=\frac{D_{\alpha}}{v_{\alpha} L}, \Theta_{\beta}=\frac{D_{\beta}}{v_{\alpha} L}, \quad V=\frac{v_{\beta}}{v_{\alpha}} .
\end{aligned}
$$

Then, equations (1) and (2) are given by,

$$
\begin{aligned}
& \frac{\partial C_{\alpha}}{\partial t}=\Theta_{\alpha} \frac{\partial^{2} C_{\alpha}}{\partial x^{2}}-\frac{\partial C_{\alpha}}{\partial x}-K_{1} C_{\alpha}+K_{2} C_{\beta}, \\
& \frac{\partial C_{\beta}}{\partial t}=\Theta_{\beta} \frac{\partial^{2} C_{\beta}}{\partial x^{2}}-V \frac{\partial C_{\beta}}{\partial x}+K_{1} C_{\alpha}-K_{2} C_{\beta} .
\end{aligned}
$$

These equations were solved numerically to obtain the distribution of solute which is equivalent to the chromatographic elution pattern.

\section{Numerical solution of state equation Difference equation \\ The chromatographic column is modelled by the lattice of time $t$ and distance $\mathrm{x}$ as shown in Fig. 1. The total number of lattice point is $\mathrm{N}$ and the value on a lattice point indicates the concentration of solute. The value on a lattice point can shift by diffusion to the adjacent points with At as shown in the figure. According to the flow rate, $C_{\alpha}$ and $C_{B}$ at the same lat- tice point move to the different points with At. The lattice was made so that after $A t$ both anomers are located exactly on the lattice points. For this purpose, the ratio of $v_{\alpha}$ and $v_{a}$ is represented by the round numbers, for instance $4: 3$, and the lattice was constructed using these numbers as illustrat- ed by Fig. 1. The mutarotation is assumed to occur only at a lattice point.}

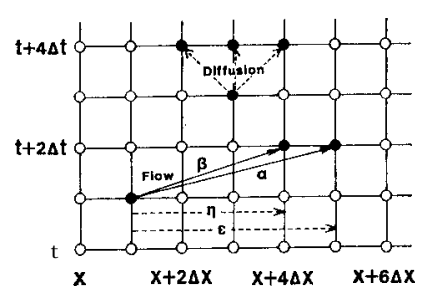

Fig. 1. Lattice model of chromatographic column. Notations: see text.

The partial derivatives with $t$ may be approximated by the difference equations as,'

$$
\begin{aligned}
& \underset{\mathrm{at}}{\mathrm{at}} \stackrel{\partial C C_{\alpha}(i, j+1)-C_{\alpha}(i, j)}{k}, \frac{}{\text { at }}, \frac{C_{\beta}(i, j+1)-C_{\beta}(i, j)}{k} \\
& k=A t \\
& i=1,2,3, \cdots, n \\
& j=1,2,3, \cdots, m
\end{aligned}
$$

where, $i$ and $\mathrm{j}$ are the numbers for distance $\mathrm{x}$ and time $t$, respectively. 
The partial derivatives of concentration with $\mathrm{x}$ may be approximated by,

$$
\begin{aligned}
& \frac{\partial C_{\alpha}}{\partial x} \rightarrow \frac{C_{\alpha}(i, j)-C_{\alpha}(i-\varepsilon, j)}{\varepsilon h}, \\
& \frac{\partial C_{B}}{\partial x} \rightarrow \frac{C_{\beta}(i, j)-C_{\beta}(i-\eta, j)}{\eta h}, \\
& \Rightarrow \frac{1 \gamma_{\alpha}}{\partial x^{2}} 2\left[\frac{C_{\alpha}(i+1, j+1)-2 C_{\alpha}(i, j+1)+C_{\alpha}(i-1, j+1)}{h_{2}}\right] \\
& \left.+\frac{C_{\alpha}(i-\varepsilon+1, j)-2 C_{\alpha}(i-\varepsilon, j)+C_{\alpha}(i-\varepsilon-1, \mathrm{j})}{h^{2}}\right], \\
& \frac{Q_{a}^{2} C_{d-1}}{\partial x^{2}} \frac{1}{2}\left[\frac{1}{2}-2 C_{B}(i, \ldots j+1)+C_{h}(i-1, \ldots j+1)\right. \\
& \left.+\frac{C_{\theta}(i-\eta+1, j)-2 C_{\beta}(i-\eta, j)+C_{\beta}(i-\eta-1, j)}{\hbar^{i}}\right] \text {, } \\
& h=\Delta x \text {, }
\end{aligned}
$$

where $\varepsilon$ and $\eta$ are the jumping width of $\alpha$-and $\beta$-anomers (see Fig. 1). With equations (6) and (7), the state equations (4) and (5) are written as,

$$
\begin{gathered}
C_{\alpha}(i, j+l)=\frac{k \Theta_{\alpha}}{2\left(h^{2}+k \Theta_{\alpha}\right)}\left[C_{\alpha}(i+1, \quad j+1)+C_{\alpha}(i-1, \quad j+1)\right. \\
\left.+C_{\alpha}(i-\varepsilon+1, j)+C_{\alpha}(i-\varepsilon-1, j)\right]+\frac{k\left(h-\varepsilon h^{2} K_{1}-\varepsilon \Theta_{\alpha}\right)}{\varepsilon\left(h^{2}+k \Theta_{\alpha}\right)} C_{\alpha}(i-\varepsilon, j) \\
+\frac{h(\varepsilon h-k)}{\varepsilon\left(h^{2}+k \Theta_{\alpha}\right)} C_{\alpha}(i, j)+\frac{k h^{2} K_{2}}{h^{2}+k \Theta_{\alpha}} C_{\beta}(i-\eta, j) \\
C_{\beta}(i, j+1)=\frac{k \Theta_{\beta}}{2\left(h^{2}+k \Theta_{\theta}\right)}\left[C_{\theta}(i+1, j+1)+C_{\theta}(i-1, j+1)\right. \\
\left.+C_{\beta}(i-\eta+1, j)+C_{\theta}(i-\eta-1, j)\right]+\frac{k\left(h V-\eta h^{2} K_{2}-\eta \Theta_{\beta}\right)}{\eta\left(h^{2}+k \Theta_{\beta}\right)} C_{\beta}(i-\eta, j) \\
+\frac{h(\eta h-k V)}{\eta\left(h^{2}+k \Theta_{\beta}\right)} C_{\beta}(i, j)+\frac{k h^{2} K_{1}}{h^{2}+k \Theta_{\theta}} C_{\alpha}(i-\varepsilon, j)
\end{gathered}
$$

Since, $C_{\alpha}(i, j)$ and $C_{\beta}(i, j)$ move by the flow to the positions, $\varepsilon h$ and $\eta h$, respectively with time-interval $k$, and do not affect the, terms, $C_{\alpha}(i, j+1)$ and $C_{\beta}(i, j+1)$. Then the equations

$$
\begin{aligned}
& \varepsilon h-k=0, \\
& \eta h-k V=0
\end{aligned}
$$

are given. The intervals, $h$ and $k$, can be estimated 'as the solutions of equation (10).

Equations (8) and (9) contain only numbers $\mathrm{j}$ and $\mathrm{j}+\mathrm{l}$ on the time-axis. For a-anomers, equation (8) can be divided into the unknown terms, $C_{\alpha}(i, j$ 
$+1), C_{\alpha}(i+1, j+1)$ and $C_{\alpha}(i-1, j+1)$, and known term $b(i)$. The similar manipulation can be applied to $\beta$-anomer. Thus, $C_{\alpha}(i, j+1)$ and $C_{\beta}(i, \mathrm{j}+1)$ for given $\mathrm{j}$-value may be represented by,

$$
\begin{aligned}
& C_{\alpha}(i, j+1)=A\left[C_{\alpha}(i+1, j+1)+C_{\alpha}(i-1, j+1)\right]+b(i), \\
& C_{\beta}(i, j+1)=E\left[C_{\beta}(i+1, j+1)+C_{\beta}(i-1, j+1)\right]+g(i), \\
& b(i)=A\left[C_{\alpha}(i-\varepsilon+1, j)+C_{\alpha}(i-\varepsilon-1, j)\right]+B C_{\alpha}(i-\varepsilon, j) \\
& +C C_{\alpha}(i, j)+D C_{\beta}(i-\eta, j) \text {, } \\
& g(i)=E\left[C_{\beta}(i-\eta+1, j)+C_{\beta}(i-\eta-1, j)\right]+F C_{\beta}(i-\eta, j) \\
& +G C_{\theta}(i, j)+H C_{\alpha}(i-\varepsilon, j) \text {, } \\
& \boldsymbol{A}=\frac{k \Theta_{\alpha}}{2\left(h^{2}+k \Theta_{\alpha}\right)}, \quad B=\frac{k\left(h-\varepsilon h^{2} K_{1}-\varepsilon \Theta_{\alpha}\right)}{\varepsilon\left(h^{2}+k \Theta_{\alpha}\right)}, \\
& C=\frac{h(\varepsilon h-k)}{\varepsilon\left(h^{2}+k \Theta_{\alpha}\right)}, D=\frac{k h^{2} K_{2}}{h^{2}+k \Theta_{\alpha}}, \\
& E=\frac{k \Theta_{\beta}}{2\left(h^{2}+k \Theta_{\beta}\right)}, \quad F=\frac{t\left(h V-\eta h^{2} K_{2}-\eta \Theta_{\beta}\right)}{\eta\left(h^{2}+k \Theta_{\beta}\right)}, \\
& G-\frac{h(\eta h-k V)}{\eta\left(h^{2}+k \Theta_{\beta}\right)}, H=\frac{k h^{2} K_{1}}{h^{2}+k \Theta_{\beta}} .
\end{aligned}
$$

Equations (11) and (12) are simultaneous algebraic equation with $n$ elements, respectively.

Numerical solution of difference equation

Equation,

$$
\frac{\mathrm{ac}}{\mathrm{at}}=\frac{\partial^{2} C}{\partial x^{2}}
$$

can be approximated by following difference equation using Crank-Nicolson method (Crank and Nicolson, 1947).

$$
\begin{aligned}
\frac{C(i, j+1)-C(i, j)}{k}= & \frac{1}{2}\left[\frac{C(i+1, j+1)-2 C(i, j+1)+C(i-1, j+1)}{h^{2}}\right. \\
& \left.+\frac{\mathrm{C}(\mathrm{i}+1, j)-2 C(i, \mathrm{j})+\mathrm{C}(\mathrm{i}-1, \mathrm{j})}{h^{2}}\right]
\end{aligned}
$$

solving with $C(i, \mathrm{j}+1)$,

$$
\begin{aligned}
& \begin{array}{l}
C(i, \mathrm{j}+1)=C(i, \mathrm{j})+\frac{\Gamma}{2} r[C(i+1, j+1)-2 C(i, j+1)+C(i-1, j+1) \\
r=\frac{k}{h^{2}}
\end{array} \\
& +C(i+1, j)-2 C(i, j)+C(i-1, j)]
\end{aligned}
$$

is obtained. When $\boldsymbol{C}(\boldsymbol{i}+1, \mathrm{j}+1), \boldsymbol{C}(\boldsymbol{i}, \boldsymbol{j}+1)$, and $\boldsymbol{C}(\boldsymbol{i}-\mathbf{1}, \mathrm{j}+1)$ are represented by $C(i+1), \mathrm{C}(\mathrm{i})$ and $C(i-1)$, respectively, the above equation is written by the general form of

$$
C(i)=\frac{1}{2} r[C(i-1)-2 C(i)+C(i+1)]+a(i) .
$$


Since equations (11) and (12) can be transformed to the form of equation (13), the iteration algorithm for solving equation (13) is also applicable to the solutions of equations (11) and (12). Let $C^{(n)}(i)$ be the value at n-l iterations starting from $C^{(1)}(i)$, the iteration formula is written by,

$$
C^{(n+1)}(i)=\frac{1}{2} r\left[C^{(n)}(i-1)-2 C^{(n)}(i)+C^{(n)}(i+1)\right]+a(i) .
$$

Before the calculation of $C^{(n+1}(i), C^{(n+1)}(i-1)$ had been already obtained. Then equation (14) is represented by,

$$
C^{(n+1)}(i)=\frac{1}{2} r\left[C^{(n+1)}(i-1)-2 C^{(n+1)}(i)+C^{(n)}(i+1)\right]+a(i) .
$$

With simple manipulation,

$$
C^{(n+1)}(i)=\frac{r}{2(1+r)}\left[C^{(n+1)}(i-1)+C^{(n)}(i+1)\right]+\frac{a(i)}{(1+r)}
$$

is obtained. This equation is further rewritten as,

$$
C^{(n+1)}(i)-\mathrm{C}^{\prime “(i)}+\left[\frac{r}{2(1+r)}\left\{C^{(n+1)}(i-1)+C^{(n)}(i+1)\right\}+\frac{a(i)}{(1+r)}-\mathrm{P}(\mathrm{i})\right],
$$

the value in the bracket is that to be revised in the $\mathrm{n}$-th and $(n+1)-\mathrm{th}$ iterations at distance i. Based upon equation (16), Young (1954) gave a modified formula which gives a large value to be revised.

$$
\begin{aligned}
& C^{(n+1)}(i)=C^{(n)}(i)+\omega\left[\frac{r}{2(1+r)}\left\{C^{(n+1)}(i-1)+C^{(n)}(i+1)\right\}+\frac{a(i)}{1+r}-C^{(n)}(i)\right], \\
& C^{(n+1)}(i)=\omega\left[\frac{r}{2(1+r)}\left\{C^{(n+1)}(i-1)+C^{(n)}(i+1)\right\}+\frac{a(i)}{1+r}\right]-(\omega-1) C^{(n)}(i),
\end{aligned}
$$

where $\omega$ is called over-relaxation parameter and given by,

$$
\left.\omega=\frac{2}{1+\left(1-\mu^{2}\right.}\right)^{1 / 2}, \quad \mu=\frac{r}{1+r} \cos \frac{\Pi}{n} .
$$

The algorithm based upon the equation is called successive over-relaxation method.

With introducing the successive over-relaxation method to equations (11) and (12),

$$
\begin{aligned}
& C_{\alpha}^{(n+1)}(i)=\omega\left[A\left\{C_{\alpha}^{(n+1)}(i-1)+C_{\alpha}^{(n)}(i+1)\right\}+b(i)\right]-(\omega-1) C_{\alpha}^{(n)}(i), \\
& C_{\beta}^{(n+1)}(i)=\omega\left[E\left\{C_{\beta}^{(n+1)}(i-1)+C_{\beta}^{(n)}(i+1)\right\}+g(i)\right]-(\omega-1) C_{\beta}^{(n)}(i), \\
& \omega=\frac{2}{1+\left(1-\mu^{2}\right)^{1 / 2}}, \mu=2 A \cos \frac{\Pi}{n}
\end{aligned}
$$

are obtained. Based upon equations (18) and (19), computer program was drawn.

\section{Boundary conditions}

To avoid the complexity of boundary conditions at the top of a partition 
column, the imaginary columns of half size in longitudinal length were added to the top and the bottom of the real column. With this imaginary column, equations (18) and (19) were used as the boundary conditions at the top of the real column without any modification.

\section{RESULTS AND DISCUSSION}

\section{Partition chromatography of GlcNAc}

The synthesized $\beta$-GlcNAc was dissolved in water at $19^{\circ} \mathrm{C}$ and allowed to stand for several hours. An aliquot was withdrawn at a suitable time and applied on a column of high-performance liquid chromatography. The elution patterns are shown in Fig. 2. The area of first peak increased gradually with incubation time. This indicated that the first peak contains predominantly $\alpha$-anomer and the second peak $\beta$-anomer. Since a separated anomer mutarotates to the other anomer, each peak became to contain a main anomer and minor other anomer, and a plateau was formed between two peaks. Thus, the height of the plateau from the base line is controlled by the rate of mutarotation and by the retention time of the anomers in the column.
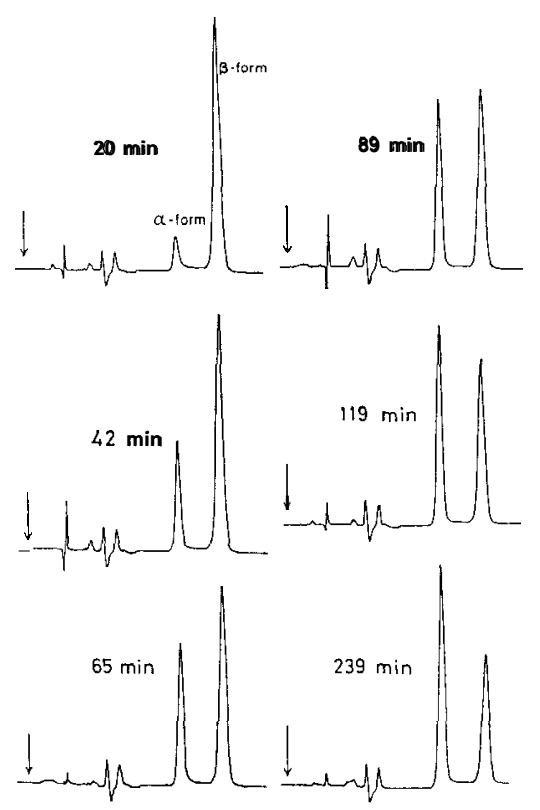

Fig. 2. Chromatographic separation of $\alpha$-and $\beta$-anomers of GlcNA . Time indicates incubation time.

\section{Factors controlling elution pattern}

In experimentally obtained elution pattern, the retention time or verti- 
cally moving rate of peaks is principally controlled by the flow rate of eluent or the pressure applied to the column, and the degree of separation of two peaks is governed by the partition coefficient between the liquid and support phases or the flow rate of each anorner. The peak shape such as the sharpness or broadness is determined by the partition coefficient, rates of diffusion in liquid and support phases and the rate of mutarotation of the anomers. The plateau height is exclusively governed by the rate constants of mutarotation, and peak-area or the ratio of peak-area of a-anomer to that of $\beta$-anomer is controlled by the ratio of both anomer in the original solution and the rate constants of the mutarotation. Thus, it is conclusively expected that the entire profile of elution pattern is determined by factors, the original ratio of concentration of $\alpha$-anomer to that of $\beta$-anomer, diffusion coefficient, flow rate of each anomer and the rate constants in mutarotation.

\section{Simulation of elution pattern}

In order to survey the effects of the values of parameters in equations (1) and (2) on the behavior of the system, the elution patterns were repeatedly calculated with changing the values of parameters, until the calculated patterns qualitatively reproduce the experimentally obtained pattern. The values of $v_{c r}$ and $v_{s}$ were determined from the experimental pattern (Fukamizo and Hayashi, 1982). The value of $c_{t \varepsilon} / c_{\beta}$ in original solution was supposed to be 1.30 , because the ratio of the solution at $\mathrm{pH} 5.0$, which allowed to stand for several hours, was found experimentally to be 1.30 . The example of calculated elution patterns is shown in Fig. 3. The values of parameters used for the calculation were $c_{\alpha} / c_{\beta}=1.30, v_{\alpha}=4.0 \mathrm{~cm} / \mathrm{min}, v_{\beta}=3.0 \mathrm{~cm} / \mathrm{min}, D_{\alpha}=D_{\beta}=$ $0.024 \mathrm{~cm}^{2} / \mathrm{min}, k_{1}=7.1 \times 10^{-3} \mathrm{~min}^{-1}, k_{2}=7.0 \times 10^{-3} \mathrm{~min}^{-1}$, and these were tentatively regarded as the standard values.

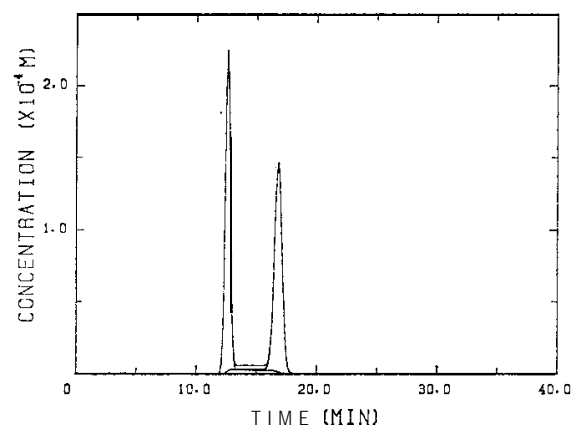

Fig. 3. Calculated elution pattern with $k_{1}+7.1 \times 10^{-3}, k_{2}=7.0 \times 10^{-3} \mathrm{~min}^{-1}$.

\section{$k_{1}-$ and $k_{2}-$ values and shape of pattern}

Under fixing other values at the standard, the elution pattern was calculated with increase in $k_{1}$-value to ten-times of the standard. The result is shown in Fig. 4. A similar calculation was performed with $k_{2}$-value twice larger than the standard and result is shown in Fig. 5. From these figures, 


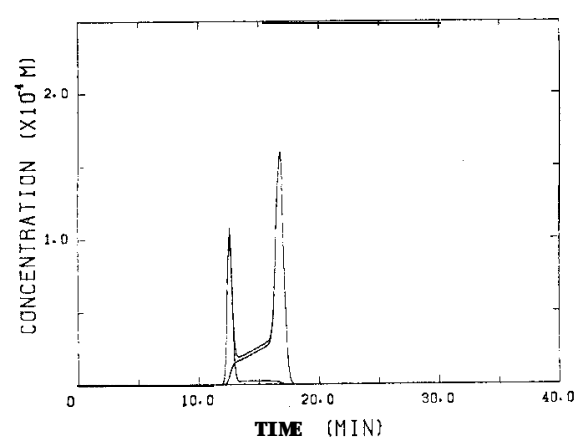

Fig. 4. Calculated elution pattern with $k_{1}=7.1 \times 10^{-2}, k_{2}=7.0 \times 10^{-3} \mathrm{~min} 1$.

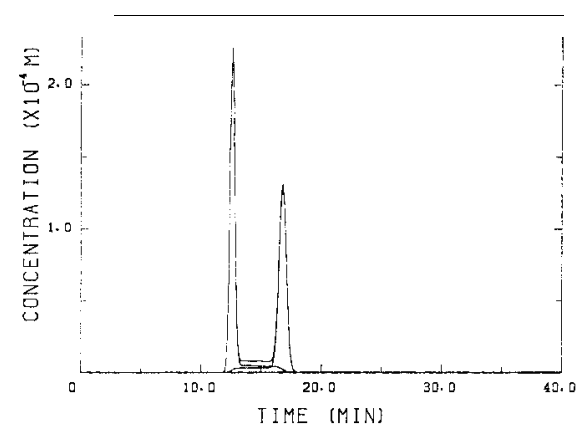

Fig. 5. Calculated elution pattern with $k_{1}=7.1 \times 10^{-3}, k_{2}=1.4 \times 10^{-2} \mathrm{~min}^{-1}$.

it is obvious that the height of the plateau between both peaks is sensitively controlled by the values of the rate constants, a large value of rate constant caused the increase in the height as expected. The ratio of $c_{\alpha} / c_{\beta}$ calculated from the patterns shown in Figs. 4 and 5 were 0.39 and 1.56 , respectively. These ratios are different profoundly from that used for calculation as an initial condition, suggesting that the ratio $c_{\alpha} / c_{\beta}$ obtained from the experimental elution pattern is not necessarily identical to that in the original solution subjected to chromatography. This problem will be discussed later at some length.

Effect of diffusion coefficient

As shown in Fig. 6, diffusion coefficient governed only the sharpness of peaks, but not the ratio $c_{\alpha} / c_{\beta}$ and the height of the plateau.

Ratio $c_{\alpha} / c_{\beta}$

As described before, the ratio $c_{\alpha} / c_{\beta}$ calculated from elution pattern may be different in some cases from that of the original solution. Therefore, it is necessary to establish a method by which the true ratio $c_{\alpha} / c_{\beta}$ is obtained from the elution pattern. The specific rotations $[\alpha]_{D}^{20}$ of pure $\beta$-GlcNAc and a-GlcNAc were reported to be -21.5 " and 82.0" (Kuhn and Haber, 1953), respectively. The aqueous solution of GlcNAc, which was allowed to 


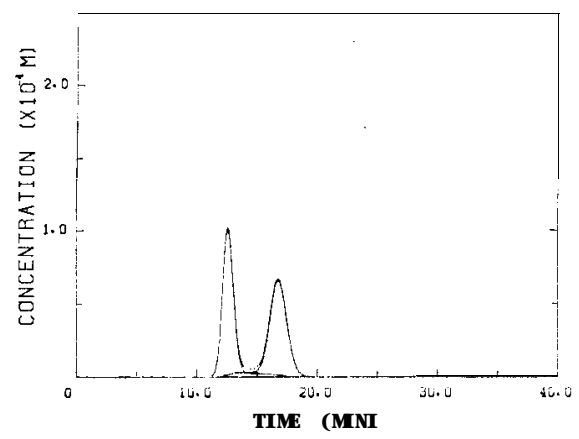

Fig. 6. Calculated elution pattern. Diffusion coefficients were 5 times as large as those used for pattern shown in Fig. 3.

stand for several days and attained to the equilibrium of mutarotation, gave $\lceil\alpha]_{D}^{20}=39.8^{\circ}$. From these values, the ratio $c_{\alpha} / c_{\beta}$ in the solution was calculated to be 1.45. On the other hand, the ratio $c_{\alpha} / c_{\beta}$ obtained from the elution pattern (not shown) of equilibrated GlcNAc solution was found to be 1.46. In elution pattern, the first peak contains a small fraction of $\beta$-anomer and reversibly the second peak contains a small fraction of a-anomer. Therefore, the good agreement of $c_{\alpha} / c_{\beta}$ in the original solution and that obtained from elution pattern appears to be coincident, and in general the ratio $\boldsymbol{c}_{\alpha} / \boldsymbol{c}_{\beta}$ in the original solution may not be estimated directly from that obtained from the elution pattern.

The elution patterns were repeatedly calculated with changing the values of diffusion coefficients and rate constant under fixing the initial condition at $c_{\alpha} / c_{\beta}=1.45$ until the calculated pattern reproduced completely the experimental pattern. With this procedure, the values of diffusion coefficients and rate constants were estimated to be $D_{\alpha}=D_{\beta}=0.024 \mathrm{~cm}^{2} / \mathrm{min}, k_{1}=5.3 \times 10^{-3} \mathrm{~min}^{-1}$ and $k_{2}=6.0 \times 10^{-3} \mathrm{~min}^{-1}$.

Next, in order to survey the relation between the ratio $c_{\alpha} / c_{\beta}$ in the original solution and that obtained from experimental elution pattern, the calculations of elution pattern were performed with changing the initial condition, the value of $c_{\alpha} / c_{\beta}$ in the original solution, using values of parameters estimated by the above procedure. The ratio $c_{\alpha} / c_{\beta}$ was obtained from the calculated elution pattern and was plotted against the ratio $c_{\alpha} / c_{\beta}$ in the original solution. The results are shown in Fig. 7. From the figure, it is obvious that when the ratios $c_{\alpha} / c_{\beta}$ in the original solution lie in the region from 1.0 to 2.0 , the $c_{\alpha} / c_{\beta}$ in the original solution (sample solution) can be directly estimated from that obtained from the experimental elution pattern. When $c_{\alpha} / c_{\beta}$ in the original solution is below 1.0 and above 2.0, some correction is necessary.

$c_{\alpha} / c_{\theta}$ and $k_{1} / k_{2}$

As described above, $k_{1}$ - and $k_{2}$-value control the ratio $c_{\alpha} / c_{\beta}$ in the elution pattern with respective and reverse modes. The elution patterns were calculated with changing the $k_{1^{-}}$and $k_{2}$-values in parallel, under fixing 


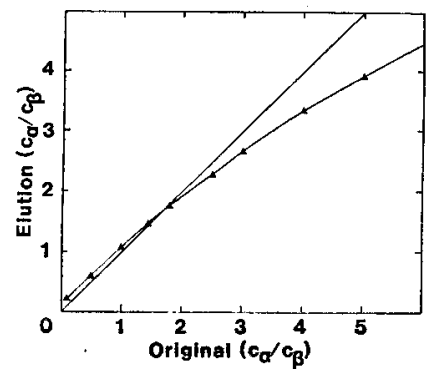

Fig. 7. Relation between molar ratio $c_{\alpha} / c_{\beta}$ of original solution and that calculated from elution pattern. When both ratios are the same, the straight fine will be obtained.

the ratio $k_{1} / k_{2}$ at $0.875\left(k_{1}=5.3 \times 10^{-3} \mathrm{~min}^{-1}, k_{2}=6.0 \times 10^{-3} \mathrm{~min}^{-1}\right.$, see the previous section) and the initial condition $c_{\alpha} / c_{\beta}=1.45$. As a result, it was found that as far as the ratio $k_{1} / k_{2}$ is fixed at a constant value, the ratio $c_{c t} / \boldsymbol{c}_{\beta}$ obtained from the elution pattern is always held at a constant value. In practical sense, the parallel change of $k_{1}$ - and $k_{2}$-value may be caused by change in milieu such as reaction temperature.

\section{General discussion}

The true diffusion coefficient of hexoses has order of $10^{-4} \mathrm{~cm}^{2} / \mathrm{min}$. The calculated values of diffusion coefficient of GlcNAc in partition column were about 100 times as large as the true value. Such a large value may be due to that diffusion coefficients defined in equations (1) and (2) contain the vertical distribution of solute arising from the partition of solute between liquid and support phases. Fukamizo and Hayashi (1982) reported that the values of $k_{1}$ and $k_{2}$ of mutarotation of GIcNAc in homogeneous $75 \%$ acetonitrile were of order of $1.5 \times 10^{-3} \mathrm{~min}^{-1}$. The values of rate constants in partition column were about four times larger than those found in $75 \%$ acetonitrile, as described above. The support phase contains water-rich medium than liquid phase does. The rate constants in $\mathrm{H}_{2} \mathrm{O}$ were found to be about 10 times larger than those in $75 \%$ acetonitrile at $19^{\circ} \mathrm{C}$. Thus, the enhanced rate of mutarotation in the support phase seems to give large values of rate constants in the partition column.

Lysozyme catalyzed the cleavage of $\beta$-1,4-glucosaminide linkage of substrate and the subsequent attack of water (hydrolysis) or nucleophile (transglycosylation) on the carbonium ion intermediate (Imoto et al., 1972). Since glycosides produced by transglycosylation were found to have only $\beta$-anomeric form, the lysozyme-catalyzed reaction has been classified as anomerretention reaction (Imoto et al., 1972). However, there were no experimental evidence on the anomeric form of reducing sugar residue which was produced by hydrolysis. Such the lack of the experimental data are due to the fact that anomeric form of produced reducing terminal mutarotates rapidly to the other anomeric form during the enzymatic reaction. In Iysozyme-catalyzed hydrolysis, an attacking water molecule may have more freedom of position 
than the bulky nucleophiles in transglycosylation. If this is the case, it is possible that some fraction of sugar residue at the reducing terminal of the nascent enzymatic product may be in $\alpha$-anomeric form. To make it clear the above question, analysis of elution pattern of the enzymatic product should be first carried out. The analytical technique and the results reported on the present paper will serve for the analysis of the lysozyme-catalyzed reaction on chitooligosaccharide substrate.

\section{REFERENCES}

Crank, J. and P. Nicolson 1947 A practical method for numerical evaluation of solutions of partial differential equations of the heat-conduction type. Proc. Cambridge Phil. Soc., 43: $50-67$

Fukamizo, T. and K. Hayashi 1982 Separation and mutarotation of chitooligosaccharides. J. Biochem., 91: 619-626

Imoto, T., L. N. Johnson, A. C. T. North, D. C. Phillips and J. A. Rupley 1972 The Enzyme, Vol. 7. Academic Press, New York (U.S.A.), pp. 665-868

Keller, R. A. and J. C. Giddings 1975 Theoretical Basis of Partition Chromatography in Chromatography, ed. by E. Heftmann, Van Nostrand Reinhold Co., New York (U.S.A.)

Kuhn, R. and F. Haber 1953 Uber die $\beta$-Form des N-Acetyllglucosamins. Chem. Ber., $86: 722-724$

Pigman, W. and E. F. L. J. Anet 1972 Mutarotations and Action of Acids and Bases in the Carbohydrates, ed. by W. Pigman and D. Horton, Academic Press, New York (U.S.A.), pp. 165

Thomas, D. and J. P. Kernevez 1976 Analysis and Control of Immobilized Enzyme Systems. North-Holland Pub. Co. (Netherlands)

Young, D. 1954 Iterative method for solving partial differential equations of elliptic type. Trans. Amer. Math. Soc., 16: 92-111 